\title{
Preparation of surface coatings on a conductive substrate by controlled motion of graphene nanoflakes in a liquid medium
}

\author{
X.F. Zhang* and R.S. Qin* \\ Department of Materials, Imperial College London, Exhibition Road, London SW7 2AZ, \\ United Kingdom
}

\begin{abstract}
Controlled motion of graphene and graphene oxide nanoflakes in a thin liquid film on metal surfaces was studied to unravel the significant variations of the electric field effects on the nanoparticles. It was found that graphene oxide flakes were negatively charged and migrated toward anode while the electrically neutral graphene flakes moved toward cathode. Therefore, thin layers of graphene as a protective coating were produced to inhibit corrosion of underlying metals and reduce friction and wear-related mechanical failures in moving mechanical systems. The method does not require an insulated substrate to confine the high electric field to the fluidic layer. The motion of the nano-particles under pulsed electric current was very efficient. The observed effects were interpreted in a possible mechanism associated to the effect of electric field on the mobility of different particles in different conductive media. This significant phenomenon, combined with unique properties of graphene and graphene oxides, represents an exciting platform for enabling diverse applications on the preparation of protective coatings on an arbitrary conductive substrate over large areas.
\end{abstract}

Keywords: Graphene; Tribological; Electrophoresis; Steel; Electric field

*Corresponding authors. Tel.: +44 (0)20 7594 6803; fax: +44 (0)20 75946757.

E-mail address: xfzhangosaka@gmail.com (X.F. Zhang), r.qin@imperial.ac.uk (R.S. Qin). 


\section{Introduction}

Reducing friction and wear-related mechanical failures in moving mechanical systems still remains a huge challenge due to friction's adverse impacts on efficiency, durability, and environmental compatibility [1-3]. Accordingly, increasing attention has been paid to develop new materials, coatings, and lubricants that can potentially provide low friction and wear even under severe operating conditions [1-3]. Graphene, a two-dimensional carbon materials, was only very recently explored that it had unusual properties to reduce wear and friction in nano-scale to macro-scale systems due to the interplay of surface traction forces [4] and the effect of electron-phonon coupling [5]. Considering the fact that most of the previous studies have concentrated on the nano-scale friction [6,7], this is of particular interest in probing the tribological behaviors of graphene at a large scale for expanded practical applications. This requires a suitable deposition method that allows the production of graphene coatings over large areas. Although the chemical vapour deposition (CVD) process is widely used to prepare a thin film, this technique can not be used in the system with large size and complex structure. Furthermore, selective growth of graphene coating on a substrate (e.g. Co, Pt, Ni, $\mathrm{Cu}$ ) also greatly affected its application [8-10]. Additionally, CVD method can not be allowed to large-scale use in industry due to high cost of its production process. Therefore, it is critical to develop a simple and effective strategy to prepare a graphene coating on an arbitrary substrate over large areas.

Previously it has been reported that the friction decreases with increasing number of layers $[5,11]$, so the coating of graphene will be produced with controlled thickness by the proposed technique. In this study, the coatings of graphene will be deposited on the steel surface by means of electrophoresis. The proposed method does not require an insulated substrate to confine the high electric field (at least $10 \mathrm{kV}$ ) [12,13] to the fluidic layer. This method can be applied to an arbitrary conductive substrate. A uniform coating with 4-6 layers can be fabricated in a few minutes. Except for reducing wear and friction, thin layers of graphene can also be as a protective coating that inhibits corrosion of underlying metals [14]. 


\section{Experiment section}

Here in the present work, pulsed electric current [15], has been implemented to drive the graphene nano-flakes in a thin ethanol film. 316L stainless steel is selected as the substrate for the thin ethanol film, which differs from the insulator substrate used in the early mentioned methods. This enables to reduce the electric potential from $\sim 10 \mathrm{kV}$ for insulator substrate to $24 \mathrm{~V}$ for conductor substrate. A commercially available ethanol solution containing graphene was obtained from Graphene Supermarket Inc. The solution was prepared by chemical exfoliation of the highly oriented pyrolytic graphite in ethanol [2]. The average diameter of graphene flakes is $550 \mathrm{~nm}$ (range: 150-3000 nm) and the average thickness is $0.35 \mathrm{~nm}$. The weight concentration of graphene was $1 \mathrm{mg} / \mathrm{L}$ containing mostly single layer graphene. Small amount solution $\left(0.1 \mathrm{~mL}\right.$ of solution per $4 \mathrm{~cm}^{2}$, that is, solution film in $0.25 \mathrm{~mm}$ thickness) was dropped on the highly polished surface of 316L stainless steel plates and the liquid ethanol part (70\% ethyl alcohol+30\% water) was evaporated in air in a few minutes. The samples were divided into two groups, with each group containing three test pieces. Group 1 was processed without pulsed electric current. Group 2 underwent exactly the same experimental configuration as Group 1 but with the addition of pulsed electric current for $4 \mathrm{~min}$. The ends of the steel plate were connected to the power supply via copper wires. The pulse is in square wave. Each pulse has a loading duration of $60 \mu \mathrm{s}$, frequency of $1 \mathrm{~Hz}$. The electric current density in steel is $1 \times 10^{7} \mathrm{~A} / \mathrm{m}^{2}$. The width between two electrodes is $20 \mathrm{~mm}$ (e.g. the width of the liquid film). The consumed electric power in pulse processing is $0.004 \mathrm{~W}$, which is much less than the power of household fluorescent. Formation of graphene deposits on the steel surface has been confirmed by optical microscope. To determine the quality and location of formed graphene film, Raman spectroscopy was employed with $532 \mathrm{~nm}$ laser.

\section{Results and discussion}

The distributions of graphene nanoparticles on the steel surface are as follows before applying the electric current. The coverage of the deposited flakes (by area) does not exceed 
$20 \%$ of the total surface area. For the deposited area (Fig.1a), the population density is relative low, which will reduce the protective properties of the coating. The chemical composition of these particle deposits was confirmed by the characteristic Raman bands (Fig.1b). In general, the Raman spectrum of single layer graphene is characterized by the sharp and large $2 \mathrm{D}$ band $\left(\sim 2700 \mathrm{~cm}^{-1}\right)$, which is the second order of the D band $(\sim 1350$ $\mathrm{cm}^{-1}$ ). The $\mathrm{D}$ peak is absent in a perfect graphene sheet because of symmetry, and grows as defects or disorders in the honeycomb structure increases [16]. The D band is the breathing mode of $\mathrm{A}_{1 \mathrm{~g}}$ symmetry, while the $\mathrm{G}$ band $\left(\sim 1580 \mathrm{~cm}^{-1}\right)$ is generally assigned to the $\mathrm{E}_{2 \mathrm{~g}}$ phonon of $\mathrm{sp}^{2}$ bonds of carbon atoms [17]. Hence, the outstanding characteristics of $2 \mathrm{D}, \mathrm{G}$, and $\mathrm{D}$ bands in Raman spectrum can be used to quantify and compare the degree of defects and disorders. Here, the defect peak D is slightly strong, suggesting that partial oxidation of graphene might have occurred during evaporation of ethanol in air. In addition, the sizes of the deposited graphene flakes are varied, but do not extend $3 \mu \mathrm{m}$ in diameter. This is in consistent with the technical parameters of the investigated particles mentioned earlier.
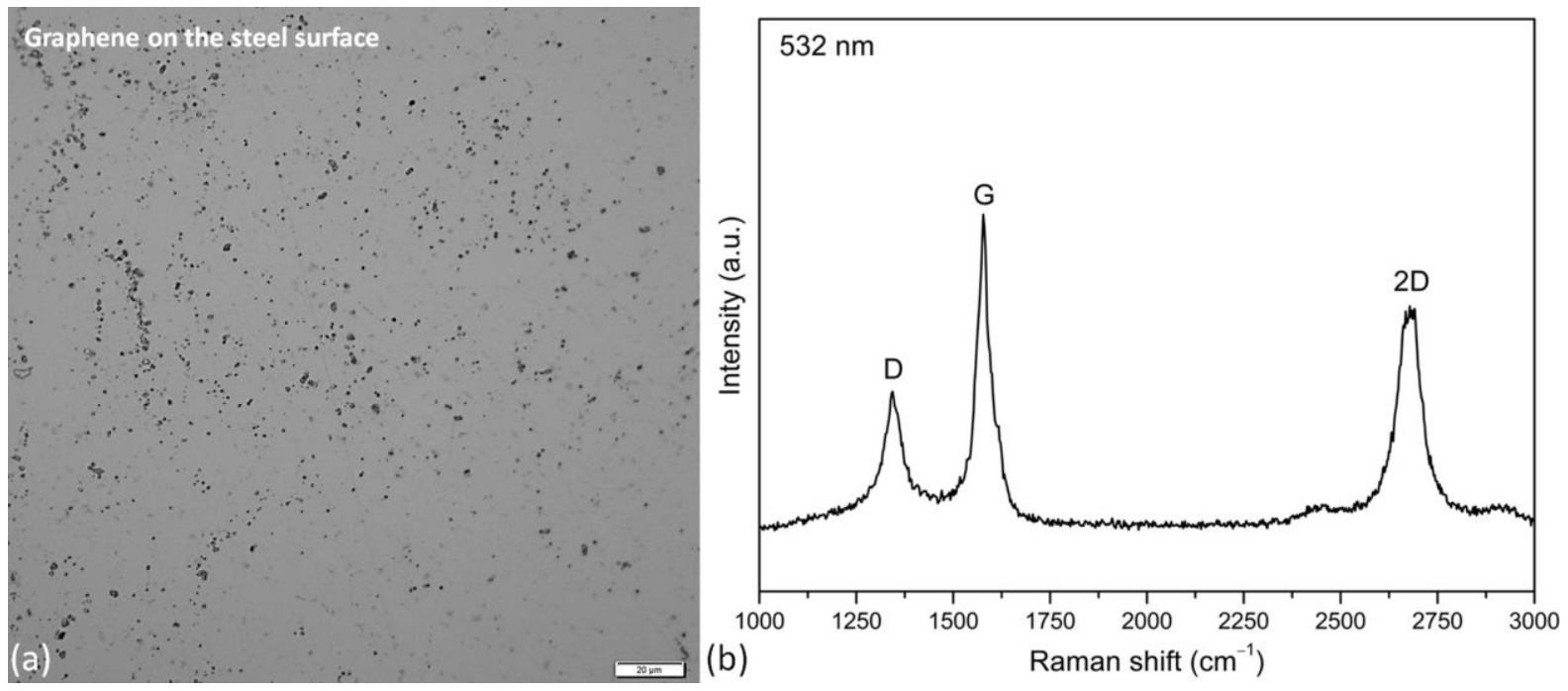

Fig.1. The distribution of graphene nanoparticles on the steel surface before applying the electric current. (a) A uniform deposit of nanoparticles (dark particles) on the steel surface after evaporation of ethanol; and (b) Raman bands were collected to characterize the characteristics of the particle deposits. 


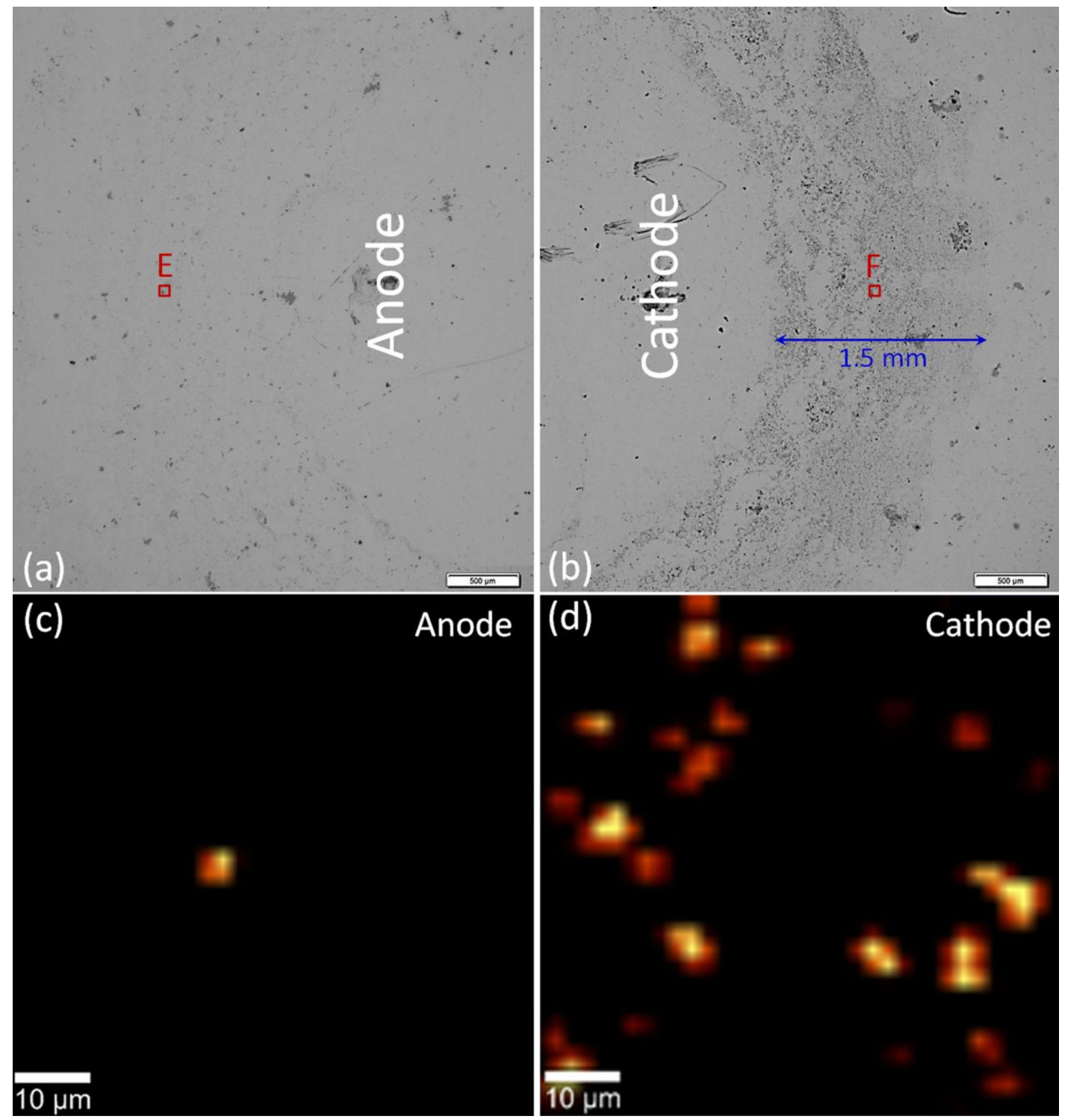

Fig.2. The distribution of nanoparticles after the application of the electric current. (a) The depostis on the anode; (b) The depostis on the cathode; (c) Two-dimensional map of the characteristic Raman peaks in selected region E on the anode; and (d) Two-dimensional map of the characteristic Raman peaks in selected region F on the cathode.

Interestingly, after the application of the electric current the distribution of nanoparticles exhibits an apparent tendency, that is, most of graphene nanoparticles migrate toward the 
cathode (Fig.2). Optical micrographs showed the formation of the deposits on the anode (Fig.2a) and the cathode (Fig.2b), respectively. An endless belt region with high density population was observed on the cathode, while a very low density area was presented on the anode. The width of the belt region on the cathode was approximately $1.5 \mathrm{~mm}$. Apart from the areas near the two electrodes, the particle aggregation cannot be observed in other regions. To analyse the spatial distribution of the deposits, two-dimensional maps of the characteristic Raman peaks were measured. Two rectangular areas with the dimensions of $70 \mu \mathrm{m} \times 70 \mu \mathrm{m}$ were selected for the Raman tests to confirm the characteristics of the deposits. The scanned results of rectangle $\mathrm{E}$ and $\mathrm{F}$ are shown in the two-dimensional maps of Fig. 2c and Fig. 2d, respectively. In the same scanned area, around 30 particles (e.g. $6122 / \mathrm{mm}^{2}$ ) were on the cathode while only one particle (e.g. $204 / \mathrm{mm}^{2}$ ) was on the anode. The size of the deposited particles was in the range of $3-12.2 \mu \mathrm{m}$ on the cathode, while the size is $4.8 \mu \mathrm{m}$ on the anode. All the sizes of the deposited particles with electric field extended $3 \mu \mathrm{m}$ in diameter. The locomotion under electric current causes aggregation of nanoparticles, thereby increasing the particle size.
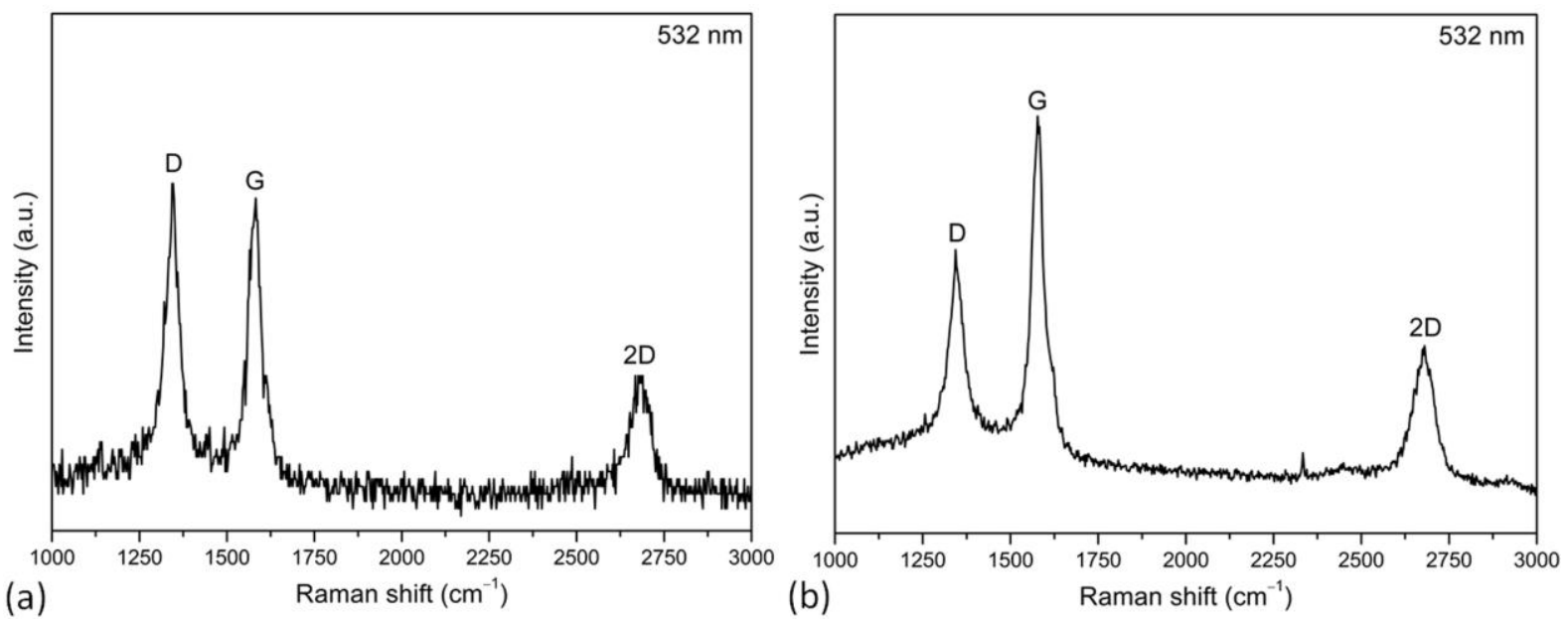

Fig.3. Raman spectra of the deposits acquired with a laser excitation wavelength of $532 \mathrm{~nm}$. (a) on the anode, and (b) on the cathode.

To analyse the number of the deposit and its defects, the intensity ratios $I_{D} / I_{G}$ and $I_{2 D} / I_{G}$ were calculated according to the Raman spectra. The band intensity ratio of $\mathrm{I}_{\mathrm{D}} / \mathrm{I}_{\mathrm{G}}$ is proportional to the number of defects, and $\mathrm{I}_{2 \mathrm{D}} / \mathrm{I}_{\mathrm{G}}$ is inversely proportional to the number of graphene layers 
$[18,19]$. The $\mathrm{I}_{\mathrm{D}} / \mathrm{I}_{\mathrm{G}}$ ratios collected at the anode (Fig.3a collected from Fig.2c) and cathode (Fig.3b collected from Fig.2d) were around 1.06 and 0.57 respectively, indicating that the defect peak D was much stronger at the anode. It suggests that graphene at the anode might be converted to a graphene oxide structure due to modifications during evaporation of ethanol in air. The different $I_{D} / I_{G}$ ratios also indicate that graphene oxides migrated toward the anode while graphene particles migrated toward the cathode. This provides a method for the separation of graphene and graphene oxide with less energy consumption. Furthermore, the $\mathrm{I}_{2 \mathrm{D}} / \mathrm{I}_{\mathrm{G}}$ ratios collected at the cathode (Fig.3b), anode (Fig.3a), and the sample without electric field (Fig. 1b) were 0.40, 0.44 and 0.69, respectively. According to previous studies by Raman spectroscopy and atomic force microscopy $[18,19]$, the number of graphene layers at cathode is determined as 6 layers $(2.1 \mathrm{~nm})$, and graphene oxide at anode consists of 4 layers $(1.4 \mathrm{~nm})$. Statistical analysis of $\mathrm{I}_{2 \mathrm{D}} / \mathrm{I}_{\mathrm{G}}$ ratio demonstrates that the average number of layers was affected by the locomotion of particles driven by the electric field. The smaller the $\mathrm{I}_{2 \mathrm{D}} / \mathrm{I}_{\mathrm{G}}$ ratio value, the more the number of layers, i.e. majority of graphene was driven toward the cathode and thereby resulting in an increase in the number of layers. Based on the above data, we can conclude that graphene oxides formed during evaporation of ethanol migrated toward the anode, but graphene nanoparticles with much more number of layers moved to the cathode. Therefore, this technique is valuable for fabrication of graphene or graphene oxide coatings with controlled thickness. Meanwhile, in order to obtain one hundred percent coverage, the anode position can be fixed, and the cathode at a rate of $1.5 \mathrm{~mm}$ per 4 min moves to the anode. For a coating of size $20 \mathrm{~mm} \times 20 \mathrm{~mm}$, it can be obtained in 14 min with $\sim 2.1 \mathrm{~nm}$ thickness by the electric current. But the coverage of the deposited flakes does not exceed $20 \%$ of the total surface area without the application of the electric current. Additionally, we may turn to the question: how to keep the nanoflakes going in a steady direction and to steer and navigate them towards their destination by temporal and spatial regulation of the speed? The answer appears to be simple because we just need to adjust pulsed current density (or voltage) and the position of the electrode. Furthermore, the particle deposition rate can also be controlled. Here, it must be noted that the graphene oxide nanosheets (e.g. the defects near the anode) can also be a good corrosion-inhibiting coating with a superfine abrasion performance, as reported in the previous studies [1-4, 20]. 
In general, it requires at least $10 \mathrm{kV}$ high voltage to drive the large-sized particles $(>200 \mu \mathrm{m})$ to move on an insulator substrate [12,13]. In this study, the voltage is only $24 \mathrm{~V}$, but it can drive the particles $(<3 \mu \mathrm{m})$ on the conductor surface. It demonstrates that the conductor substrate can reduce required electric field value (at least reduced by more than 400 times) greatly and has a huge contribution to the movement of small particles. Moreover, if the objects are very small (typically in the micron or nanoscale), viscosity effects will become very important and therefore momentum-based propulsion (e.g. bubble propulsion produced by water-splitting or other redox reactions) is increasing difficult [12]. The friction that the particle has to overcome in order to move is amplified, due to the disadvantageous evolution of the surface to volume ratio. The particles are more affected by Brownian motion and therefore more force has to be applied. As a direct consequence, very high external electric fields in the MV range are necessary if one wants to trigger on a water-splitting reaction on objects with a size in the nano-sized range [12]. Therefore, bubble propulsion is not playing a significant role in this study. Additionally, the force induced by surface tension is negligible because surface tension change does not occur [12].

A possible mechanism has been suggested for the observed separation of the nanoparticles in a thin liquid film on top of a conducting substrate. For simplicity (Fig.4), there are two different conductive media in a steady current system, one with electrical conductivity $\sigma_{1}$ and another with $\sigma_{2}$. The basic equations are given in the following expressions:

$$
\begin{aligned}
& \nabla \cdot \vec{J}=0 \\
& \nabla \times \vec{E}=0
\end{aligned}
$$

where $J$ is electric current density, $E$ is electric field intensity, and $\nabla$ is Laplace operator. Boundary conditions on the interface between conductive medium $\sigma_{1}$ and $\sigma_{2}$ can be obtained from Eq. (1) as

$$
\begin{aligned}
& \vec{n} \times\left(\vec{E}_{2}-\vec{E}_{1}\right)=0 \\
& \vec{n} \times\left(\vec{J}_{2}-\vec{J}_{1}\right)=0
\end{aligned}
$$

where $\vec{n}$ is normal vector. By application of boundary conditions, the law of refraction on the interface can be derived as follows

$$
\frac{\tan \theta_{1}}{\tan \theta_{2}}=\frac{\sigma_{1}}{\sigma_{2}}
$$


Here, the electrical conductivity of the steel $\left(\sigma_{1}\right)$ is equal to $10^{6} \Omega^{-1} \mathrm{~m}^{-1}$ and that of ethanol solution $\left(\sigma_{2}\right)$ is approximately $10^{-6} \Omega^{-1} \mathrm{~m}^{-1}$. The value of the ethanol solution is $10^{12}$ times smaller than that of the steel. Therefore, the Eq. (3) can be written as: $\tan \theta_{2} \approx 10^{-12} \tan \theta_{1}$. When $\sigma_{1}>>\sigma_{2}, \theta_{2} \approx 0$ if $\theta_{1} \neq \pi / 2$. Then, we can conclude that electric filed lines are approximately perpendicular to the interface.

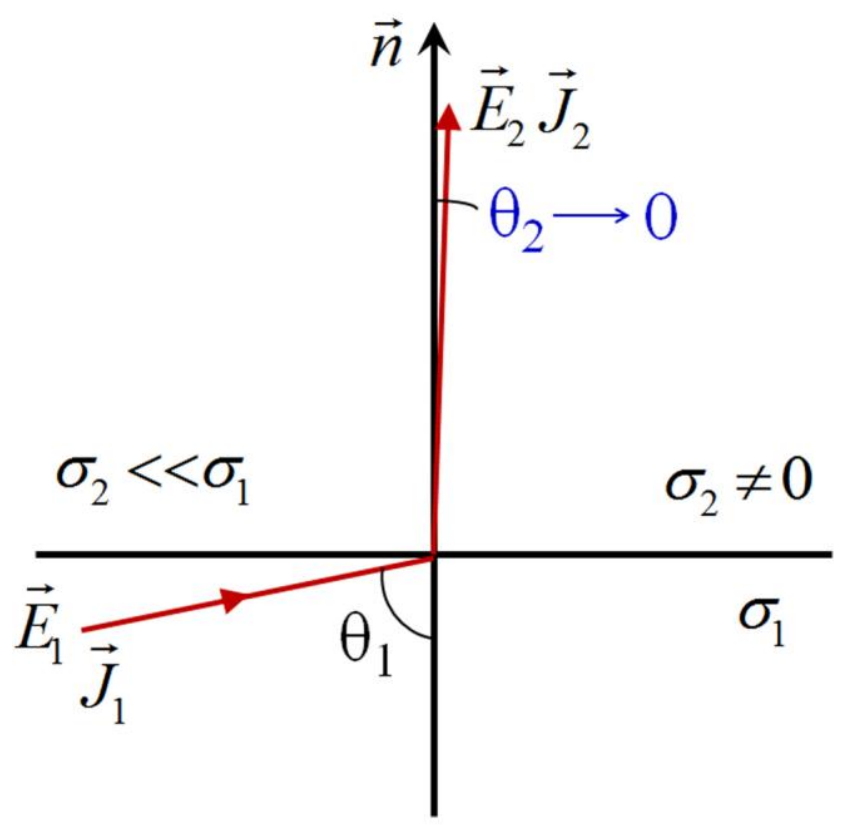

Fig.4. Schematic diagram demonstrates electric current and electric field on the different conductive media in a steady current system, one with electrical conductivity $\sigma_{1}$ and another with $\sigma_{2}$.

For the graphene oxide, it normally carries a negative charge $(\mathrm{pH}>4)$ due to the dissolution of carboxylic groups [13]. Under an electric field (Fig.5a), there are two forces acting on the graphene oxides, the electric force and the hydrodynamic force (e.g. flow friction). Thus, the suspended graphene oxide particles will migrate toward the interface between steel and ethanol solution driven by electric force. Usually, graphene oxides have higher electrical resistivity (lower conductivity) than that of the metal; thus the locations of the particles will affect the distribution of electric current [15]. When the negative particles reach the interface, the current density inside the particles is lower than that of the outside, which will increase the system free energy of the particles. In order to minimize the free energy, the particles will 
be driven to move. To achieve a directional motion to anode (Fig.5b), the electric force exerted upon the particles overcame the retardation effects, including the hydrodynamic force between the particle and its surrounding ethanol solution as well as the frictional force between the particle and the surface of the steel substrate. Due to the movement of graphene oxides toward anode, a counter flow will drive fluid and particulate motion in the negative direction (Fig.5c). When the electricity was switched off after $4 \mathrm{~min}$, it was found that graphene oxide particles were negatively charged and migrated to the positive direction while the electrically neutral graphene particles were toward the negative direction (Fig.5d), indicating significant variations of the electric current effects on the nanoparticles. For this technique, it enables to reduce the electric potential from $\sim 10 \mathrm{kV}$ for insulator substrate to $24 \mathrm{~V}$ for conductor substrate. In other word, the electric field strength required to separate the graphene nanoflakes with average diameter of $550 \mathrm{~nm}$ (range: 150-3000 nm) to be $24 \mathrm{~V}$. It demonstrates that the conductor substrate can reduce required electric field value greatly and has a huge contribution to the movement of small particles.

In the normal use of electroosmotic flow or electrophoresis, it tends to be related to the relatively high applied potentials that are required to generate significant flow velocities $[12,13]$. The use of these relatively high potentials also results in conditions of significant ohmic energy conversion generally referred to as Joule heating. This effect can result in temperature variations within a channel network, thereby leading to significant variations in local viscosity, uneven thermal conditions for chemical reactions, and potentially culminating in localized in-channel boiling. In this study, the surface temperatures of all the investigated samples were calibrated using thermocouple and were confirmed to be the same (room temperature). The temperature rising ( $\Delta T$ ) caused by ohmic heat after each pulse is obtainable from $\Delta T=j^{2} \rho_{e} \Delta t /\left(c \rho_{d}\right)$ [21], where $j$ is current density, $\rho_{e}$ is the electrical resistivity, $\Delta t$ is the pulse duration, $c$ is the specific heat of steel, and $\rho_{d}$ is the density of steel. Substituting the parameters implemented in the experiments, i.e. $j=1 \times 10^{7} \mathrm{~A} / \mathrm{m}^{2}, \rho_{\mathrm{e}}=7.40 \times 10^{-7}$ $\Omega \cdot \mathrm{m}, \Delta \mathrm{t}=60 \mu \mathrm{s}=6 \times 10^{-5} \mathrm{~s}, \mathrm{c}=500 \mathrm{~J} / \mathrm{kg} \cdot \mathrm{K}$ and $\rho_{\mathrm{d}}=7.8 \times 10^{3} \mathrm{~kg} / \mathrm{m}^{3}$, one has $\Delta \mathrm{T}=0.001 \mathrm{~K}$. The frequency of electropulsing is $1 \mathrm{~Hz}$. This means the heating rate due to ohmic heat is 0.001 $\mathrm{K} / \mathrm{s}$. This heating rate can be negligible, so the surface temperatures of all the investigated samples are the same. More importantly, this method does not require a highly insulating 
substrate to confine the electric field to the fluidic channel, thereby greatly reducing the ohmic heat generation. Furthermore, the formation of coatings on some conductive substrates (such as $\mathrm{Cu}, \mathrm{Al}, \mathrm{Ti}, \mathrm{Ni}, \mathrm{Mg}$ ) also demonstrated the feasibility of this method for preparing a large-area film.
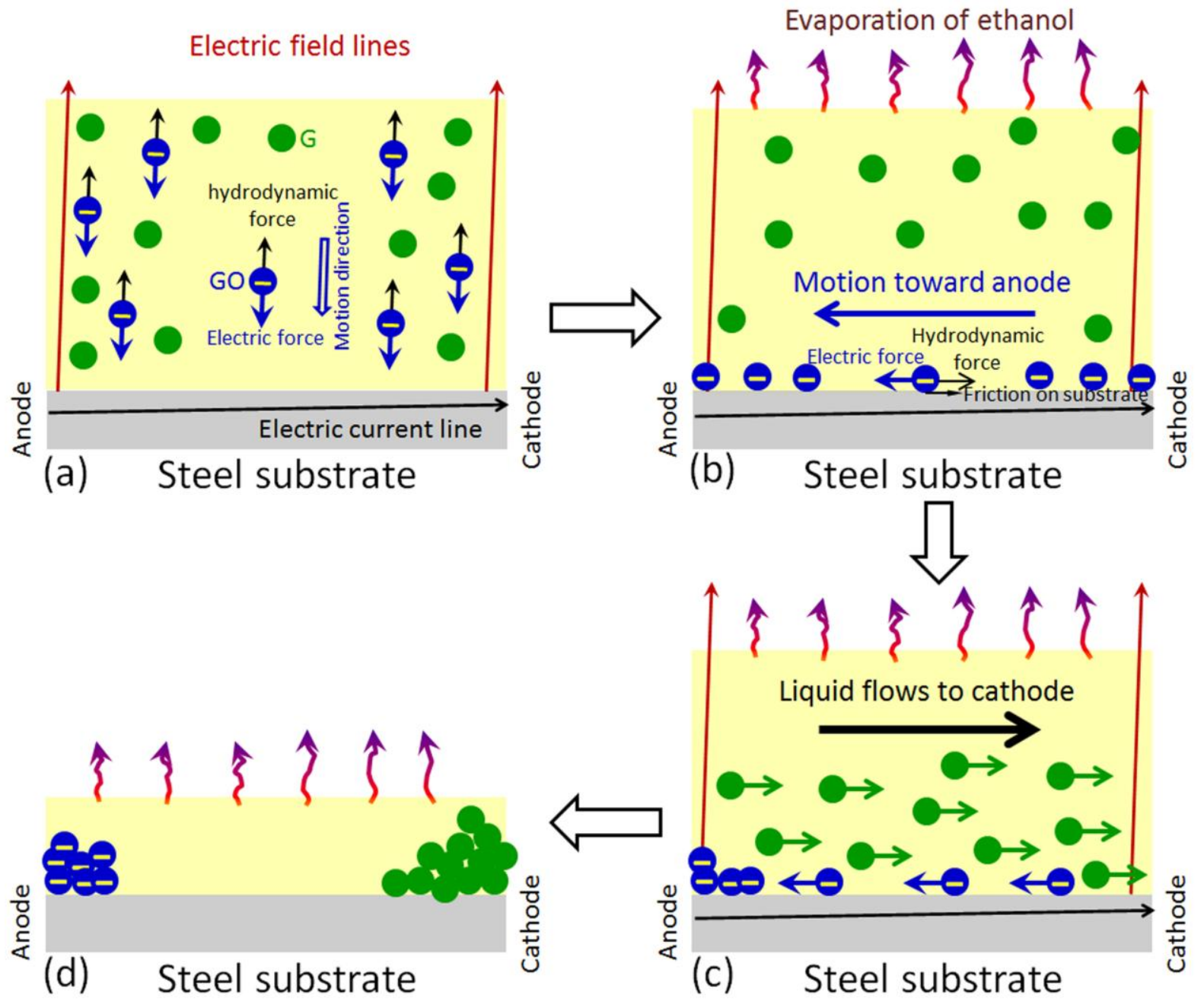

Fig.5. Schematic illustrations of nanoparticles motion toward the electrodes. Graphene oxides particles (a) firstly migrated toward the interface between steel and ethanol solution, and then (b) migrated toward the anode; (c) Graphene migrated toward the cathode; and (d) A cumulative graphene and graphene oxide respectively on the cathode and anode.

\section{Conclusions}

In conclusion, externally triggered motion of nanoparticles has been demonstrated on the steel surface under pulsed electric current. As the graphene oxide particles are negatively 
charged, the migration toward anode is induced by electric field. This in turn leads to a directional motion of the electrically neutral graphene particles toward the negative direction. Such a phenomenon has a potential application in fabrication of graphene coatings on an arbitrary conductive substrate over large areas.

\section{Acknowledgments}

The authors gratefully acknowledge the financial support from Royal Academy of Engineering and EPSRC (No. EP/J011460/1) at United Kingdom.

\section{References}

[1] D. Berman, A. Erdemir, A.V. Sumant, Materials Today 17 (2014) 31-42.

[2] D. Berman, A. Erdemir, A.V. Sumant, Carbon 59 (2013) 167-175.

[3] M.S. Won, O.V. Penkov, D.E. Kim, Carbon 54 (2013) 472-481.

[4] H. Lee, N. Lee, Y. Seo, J. Eom, S. Lee, Nanotechnology 20 (2009) 325701.

[5] T. Filleter, J.L. McChesney, A. Bostwick, E. Rotenberg, K.V. Emtsev, Th. Seyller, K. Horn, R. Bennewitz, Phys. Rev. Lett. 102 (2009) 086102.

[6] C.G. Lee, X.D. Wei, J.W. Kysar, J. Hone, Science 321 (2008) 385-388.

[7] C. Gómez-Navarro, M. Burghard, K. Kern, Nano Lett. 8 (2008) 2045-2049.

[8] R.J. Young, I.A. Kinloch, L. Gong, K.S. Novoselov, Composites Sci. Technol. 72 (2012) 1459-1476. 
[9] M. Taghioskoui, Materials Today 12 (2009) 34-37.

[10] C. Mattevi, H. Kim, M. Chhowalla, J. Mater. Chem. 21 (2011) 3324-3334.

[11] C.G. Lee, Q.Y. Li, W. Kalb, X.Z. Liu, H. Berger, R.W. Carpick, J. Hone, Science 328 (2010) 76-80.

[12] G. Loget, A. Kuhn, Nat. Commun. 2 (2011) 535-540.

[13] J.J. Zhao, G.F. Chen, W. Zhang, P. Li, L. Wang, Q.L. Yue, H.S. Wang, R.X. Dong, X.L. Yan, J.F. Liu, Anal. Chem. 83 (2011) 9100-9106.

[14] D. Prasai, J.C. Tuberquia, R.R. Harl, G.K. Jennings, K.I. Bolotin, ACS Nano 6 (2012) 1102-1108.

[15] X.F. Zhang, and R.S. Qin, Appl. Phys. Lett. 104 (2014) 114106.

[16] S.K. Jerng, D.S. Yu, J.H. Lee, C. Kim, S. Yoon, S.H. Chun, Nanoscale Res. Lett. 6 (2011) 565-571.

[17] O. Akhavan, ACS Nano 4 (2010) 4174-4180.

[18] L.Y. Lin, D.E. Kim, W.K. Kim, S.C. Jun, Surf. Coat. Technol. 205 (2011) 4864-4869.

[19] A.C. Ferrari, Solid State Commun. 143 (2007) 47-57.

[20] K. Krishnamoorthy, A. Ramadoss, S.J. Kim, Sci. Adv. Mater. 5 (2013) 406-410.

[21] R.S. Qin, B.L. Zhou, Int. J. Non Equilib. Process 11 (1998) 77-86. 